\title{
Letter
}

\section{Questioning the consensus on placebo and nocebo effects}

\author{
Doug Hardman ${ }^{\mathrm{a}}$, Phil Hutchinson ${ }^{\mathrm{b}}$, Giulio Ongaro ${ }^{\mathrm{c}}$
}

a Department of Psychology, Bournemouth University, UK
${ }^{\mathrm{b}}$ Department of Psychology, Manchester Metropolitan University, UK
${ }^{\mathrm{c}}$ Department of Anthropology, London School of Economics and Political Science, UK

Short Title: Placebo and nocebo effects

Corresponding Author:

Doug Hardman

Department of Psychology

Bournemouth University

Poole House, Talbot Campus

Fern Barrow

Poole

BH12 5BB

Tel: +44 (0)1202965274

E-mail: dihardman@bournemouth.ac.uk

Number of Tables: 0

Number of Figures: 0

Word count: 461 
We read with interest a recent article in this journal reporting the consensus of 27 experts on what should be communicated to patients about placebo and nocebo effects and how clinicians should be trained to deliver this information. ${ }^{1}$ The authors propose that communicating general information to patients about placebo and nocebo effects is beneficial but should be adjusted to context. They further propose that training clinicians to communicate about placebo and nocebo effects should be a regular and integrated part of medical education. These recommendations build on an earlier consensus statement regarding maximizing placebo effects and minimizing nocebo effects in clinical practice. ${ }^{2}$ In response, we argue that the latest consensus statement is conceptually ambiguous and does not accord with recent research on the views of patients and clinicians. Furthermore, the presentation of these consensus statements belies lively debates and disagreements in placebo studies research, including on fundamental issues such as the dominance of cognitivist accounts of placebo and nocebo effects. ${ }^{3,4}$

As the authors note, their method did not allow them to draw conclusions about specific strategies that can maximize placebo effects and minimize nocebo effects. This is, of course, because 'placebo' and 'nocebo' are merely umbrella terms that, although useful for coordinating research, encompass a diverse array of situation-dependent practices too numerous to mention; practices that patients and clinicians engage in and talk about without need for the abstract umbrella terms. As a previous editorial position of this journal on the clinical inadequacy of the placebo model suggests ${ }^{5}$, the attempt to offer general guidelines and training on placebo and nocebo effects risks obscuring what can be better communicated more precisely. 6,7

Conceptual concerns notwithstanding, recommending that tailored, evidence-based explanations of placebo and nocebo effects should be explained to patients - and that the terms themselves are acceptable - is at odds with recent systematic reviews of the use and understanding of clinical placebo effects. For example, one qualitative synthesis of 28 studies in primary care concluded that there is so much disconnect between modern scientific definitions of placebo effects and how patients and clinicians understand them, that attempts to bridge this gap are unlikely to succeed..$^{8}$ This not only undermines potential communication and training strategies, but also questions existing prevalence of use data and broader empirical findings on placebo effects in clinical practice. ${ }^{8,9}$ Moreover, although the recommendation for guidelines and training in communicating about placebo and nocebo effects is well-intentioned, given the unmanageable number of existing guidelines in modern evidence-based medicine ${ }^{10}$, it is unlikely that clinicians will have time to 
34 cases probably nothing. In most cases - except certain specific scenarios - there are likely less

35 confusing and contentious ways in which to talk about phenomena the umbrella terms purport to 36 encompass. 


\section{Statements}

\section{Conflict of Interest Statement}

40 The authors have no conflicts of interest to declare.

41 Author Contributions

42 Doug Hardman: Conceptualization; Formal analysis; Writing - original draft.

43 Phil Hutchinson: Conceptualization; Formal analysis; Writing - review \& editing.

44 Giulio Ongaro: Conceptualization; Formal analysis; Writing - review \& editing. 


\section{References}

1. Evers AWM, Colloca L, Blease C, et al. What Should Clinicians Tell Patients about Placebo and Nocebo Effects? Practical Considerations Based on Expert Consensus. Psychotherapy and Psychosomatics 2020.

2. Evers AWM, Colloca L, Blease C, et al. Implications of Placebo and Nocebo Effects for Clinical Practice: Expert Consensus. Psychotherapy and Psychosomatics 2018: 1-7.

3. Thompson JJ, Ritenbaugh C, Nichter M. Reconsidering the placebo response from a broad anthropological perspective. Culture, Medicine, and Psychiatry 2009; 33(1): 112-52.

4. Hutchinson P. The "placebo" paradox and the emotion paradox: Challenges to psychological explanation. Theory \& Psychology 2020; 30(5): 617-37.

5. Fava GA, Guidi J, Rafanelli C, Rickels K. The Clinical Inadequacy of the Placebo Model and the Development of an Alternative Conceptual Framework. Psychotherapy and Psychosomatics 2017; 86(6): 332-40.

6. Turner A. 'Placebos' and the logic of placebo comparison. Biology \& Philosophy 2012; 27(3): 419-32.

7. Moerman DE. Chapter 18 - Against 'Placebo.' The Case for Changing our Language, and for the Meaning Response. In: Colloca L, Flaten MA, Meissner K, eds. Placebo and Pain. San Diego: Academic Press; 2013: 183-8.

8. Hardman D, Geraghty AWA, Lewith G, Lown M, Viecelli C, Bishop FL. From substance to process: A meta-ethnographic review of how healthcare professionals and patients understand placebos and their effects in primary care. Health 2018; 24(3): 315-40.

9. Linde K, Atmann O, Meissner K, et al. How often do general practitioners use placebos and non-specific interventions? Systematic review and meta-analysis of surveys. PLoS One 2018; 13(8): e0202211.

10. Greenhalgh T, Howick J, Maskrey N. Evidence based medicine: a movement in crisis? BMJ 2014; 348: g3725. 